Abstract 276 Table 1 Univariate regression analysis for outcome of LN patients treated with prednisone+ pulse CTX therapy

\begin{tabular}{|c|c|c|c|c|}
\hline & $\begin{array}{l}\mathrm{CR} \\
(\mathrm{n}=54)\end{array}$ & $\begin{array}{l}\mathrm{PR}+\mathrm{NR} \\
(\mathrm{n}=41)\end{array}$ & $\begin{array}{c}P \\
\text { value }\end{array}$ & OR (95\%RI) \\
\hline Age, years & $33.78 \pm 10.75$ & $33.00 \pm 12.50$ & 0.745 & $1.006(0.971,1.043)$ \\
\hline Gender(male/female) & $6 / 48$ & $3 / 38$ & 0.399 & $0.632(0.148,2.692)$ \\
\hline Duration of disease, months & $59.60 \pm 64.71$ & $62.98 \pm 52.81$ & 0.786 & $0.999(0.992,1.006)$ \\
\hline $\mathrm{Ccr}, \mathrm{ml} / \mathrm{min}^{*}$ & $121.85 \pm 37.65$ & $106.11 \pm 42.08$ & 0.058 & $1.010(0.999,1.021)$ \\
\hline $\mathrm{ESR}, \mathrm{mm} / \mathrm{h}$ & $36.56 \pm 23.12$ & $38.75 \pm 26.12$ & 0.995 & $1.000(0.984,1.016)$ \\
\hline \multicolumn{5}{|l|}{ SLEDAI } \\
\hline total score & $10(6,26)$ & $12(4,18)$ & 0.308 & $1.060(0.948,1.185)$ \\
\hline renal score & $4(4,16)$ & $4(4,12)$ & 0.967 & $1.003(0.853,1.180)$ \\
\hline Activity Index & $6(1,15)$ & $5(1,12)$ & 0.738 & $0.979(0.863,1.110)$ \\
\hline Chronicity Index & $2(0,6)$ & $2(0,6)$ & 0.392 & $0.899(0.706,1.147)$ \\
\hline \multicolumn{5}{|l|}{ Pathological type(\%) } \\
\hline With Class III/V & $48(88.9)$ & $33(80.5)$ & 0.258 & $1.939(0.616,6.110)$ \\
\hline Class $V$ & $29(53.7)$ & $25(61.0)$ & 0.479 & $0.742(0.325,1.693)$ \\
\hline Renal p62 low expression(\%)* & $35(64.8)$ & $13(31.7)$ & 0.002 & $0.252(0.106,0.597)$ \\
\hline Low serum C3 (\%) & $50(92.6)$ & $35(85.4)$ & 0.521 & $2.143(0.563,8.158)$ \\
\hline Serum $\operatorname{lgG}, g / L^{*}$ & $12.20 \pm 5.06$ & $10.09 \pm 6.03$ & 0.089 & $1.077(0.989,1.172)$ \\
\hline Anti-dsDNA antibody, IU/m|* & $63.27 \pm 40.663$ & $49.06 \pm 39.01$ & 0.094 & $1.009(0.998,1.020)$ \\
\hline \multicolumn{5}{|l|}{ ENA $(\%)$} \\
\hline Anti-Sm* & $7(13.7)$ & $12(30)$ & 0.058 & $0.371(0.130,1.056)$ \\
\hline Anti-SSA & $27(52.9)$ & $19(47.5)$ & 0.606 & $1.243(0.543,2.849)$ \\
\hline Anti-SSB & $12(23.5)$ & $5(12.5)$ & 0.180 & $2.154(0.690,6.726)$ \\
\hline Anti-RNP & $19(37.3)$ & $18(45)$ & 0.455 & $0.726(0.312,1.686)$ \\
\hline Anti-nucleosome antibody & $32(65.3)$ & $22(59.5)$ & 0.579 & $1.283(0.532,3.098)$ \\
\hline Anti-ribosomal antibody & $5(9.8)$ & $8(20)$ & 0.168 & $0.435(0.130,1.451)$ \\
\hline \multicolumn{5}{|l|}{ Medication } \\
\hline Methylprednisolone $_{\max } \mathrm{mg} / \mathrm{day}$ & $40(8,500)$ & $40(4,200)$ & 0.584 & $1.002(0.994,1.010)$ \\
\hline Antimalarial drug $(\%)^{*}$ & $45(83.3)$ & $27(65.9)$ & 0.053 & $2.593(0.989,6.797)$ \\
\hline ACEI/ARB $(\%)$ & $26(48.1)$ & $19(46.3)$ & 0.861 & $1.075(0.477,2.425)$ \\
\hline
\end{tabular}

Background Previous studies found autophagy contributes to the pathogenesis of systemic lupus erythematosus (SLE). Whether autophagy is involved in lupus nephritis (LN) is not elucidated. P62 is a specific substrate that is degraded through autophagy-lysosomal pathway.

Methods Immunohistochemistry staining was performed to evaluate expressions of p62 in the biopsy kidney tissue of LN patients $(n=128)$ and normal control $(n=6)$. One hundred and five patients were given prednisone +CTX pulse therapy as induction treatment and followed by 24 weeks. Clinicopathologic features and induction phase remission efficacy were recorded and correlated with renal p62 expression level.

Results Compared with the controls, the expression of p62 was significantly decreased in LN biopsy tissues $(p=0.0013)$, suggesting increasing autophagy in LN kidney. However, patients with low expression of p62 had less severe nephritis, showing significantly less proteinuria, fewer interstitial fibrosis score and higher estimated creatinine clearance rates ( $p=0.0122, \quad p=0.0048, \quad p=0.0231$, respectively). Logistic regression analysis revealed that lower renal p62 expression was an independent factor associated with achieving complete remission $(\mathrm{CR})$ in 24 weeks $(\mathrm{p}=0.025)$. Patients with low $\mathrm{p} 62$ were more likely and quicker to achieve CR (Person ChiSquare test, $\mathrm{p}=0.001$; Kaplan-Meier test, $\mathrm{p}=0.0294)$.

Conclusions Low renal p62 expression was associated with less severe lupus nephritis and better short-time outcome. Because low p62 expression is the result of high level of autophagy, this data suggested that autophagy might play a protective role in LN kidney. More studies are needed to evaluate the role autophagy plays in multiple organs and cell subtypes in SLE.

Funding Source(s): None

\section{BODY MASS INDEX AT TIME OF DIAGNOSIS IS PREDICTIVE OF FUTURE DISEASE ACTIVITY IN SLE}

${ }^{1}$ George Stojan*, 2 Laurence Magder, ${ }^{3}$ Daniel Goldman, ${ }^{3}$ Michelle Petri. ${ }^{1} J o h n s$ Hopkins University; ${ }^{2}$ University of Maryland; ${ }^{3}$ Johns Hopkins University School of Medicine
Background Obesity is more common in patients with SLE compared to the general population. The prevalence of obesity among SLE patients is between 28 and 50 percent. We previously demonstrated an inverse correlation between body mass index and disease activity in systemic lupus, even after adjusting for prednisone dose. The question of whether these findings were epiphenomena related to disease activity itself remained unanswered. We thus hypothesized that the BMI at cohort entry was predictive of future disease activity.

Methods 2406 patients in a prospective SLE cohort had their weight assessed at cohort entry. Patients were categorized into five predetermined groups based on weight: low (BMI $<20 \mathrm{~kg} / \mathrm{m} 2$ ), normal weight (reference, BMI 20$24.9 \mathrm{~kg} / \mathrm{m} 2)$, overweight $(25-29.9 \mathrm{~kg} / \mathrm{m} 2)$, obese (BMI 30$34.9 \mathrm{~kg} / \mathrm{m} 2$ ), and severely obese (BMI $>35 \mathrm{~kg} / \mathrm{m} 2$ ). Disease activity was characterized on the basis of SLE Disease Activity Index (SLEDAI) scores; for each BMI category we created an Adjusted Mean SLEDAI score. This involved calculating the area under the curve of SLEDAI scores over time. The area under the curve between each two visits was the average of the SLEDAI values at those two visits multiplied by the length of time between the two visits. All the calculated areas were then summed and divided by the total length of the time period. The adjusted mean SLEDAI had the same units as SLEDAI. The adjusted mean SLEDAI has been shown to be a valid measure of SLE activity. To calculate adjusted mean of SLEDAI over time, we only included patients attending the clinic at 3 month intervals for a minimum of 3 visits. 1896 patients were included in the analysis. 1763 (93.0\%) were females. Majority (53.0\%) were Caucasians, 39.0\% African American. To determine whether BMI at cohort entry was predictive of future disease activity, we compared the categorized BMI at cohort entry with the adjusted mean SLEDAI by pairwise $t$ test and ANOVA test.

Results See table 1.

Conclusions Body weight at cohort entry was predictive of future disease activity. Overweight and obese patients had a significantly lower adjusted mean SLEDAI over time $(p<0.05)$. 


\begin{tabular}{|c|c|c|c|}
\hline BMI & $\mathbf{N}(\%)$ & SLEDAI Mean (SD) & $P$ value \\
\hline Low (reference) & $183(9.7)$ & $2.9(2.2)$ & REF \\
\hline Normal & $655(34.5)$ & $2.7(2.4)$ & 0.3107 \\
\hline Overweight & $531(28)$ & $2.5(2.1)$ & 0.0285 \\
\hline Obese & $290(15.3)$ & $2.6(2.1)$ & 0.0285 \\
\hline Severely Obese & $237(12.5)$ & $2.5(2.1)$ & 0.0587 \\
\hline
\end{tabular}

This analysis adds further support to the existence of an obesity paradox in SLE.

Funding Source(s): The Hopkins Lupus Cohort was funded by AR 69572

\section{STUDY ON SERUM DNAASE1 ACTIVITY IN PEDIATRIC ONSET SYSTEMIC LUPUS ERYTHEMATOSUS FROM A TERTIARY CARE CENTRE IN NORTH WEST INDIA}

${ }^{1}$ Nameirakpam Johnson*, ${ }^{2} \mathrm{~N}$ Ramalingeswara, ${ }^{3}$ Amit Rawat, ${ }^{4}$ Deepti Suri, ${ }^{4}$ Surjit Singh. ${ }^{1}$ Dept. of Pediatrics, Allergy- Immunology Unit, Postgraduate Institute of Medical Education and Research; ${ }^{2}$ Advanced Pediatrics Centre, Postgraduate Institute of Medical Education and Research; ${ }^{3} P G I M E R$ Chandigarh; ${ }^{4}$ Postgraduate Institute of Medical Education and Research, Chandigarh, India

\subsection{6/lupus-2019-Ism.278}

Background DNAase is an apoptotic endonuclease responsible for degradation of chromatin released by inappropriately cleared dead cells. DNAase1 activity in systemic lupus erythematosus (SLE) patients is lower than that in inactive disease in studies conducted in adult SLE patients from developed country. There is a paucity of data on DNAase1 activity in paediatric SLE from India.

Methods A cross-sectional observational study was conducted over a period of 1 year. Thirty-three consecutive children with pediatric-onset SLE were enrolled and divided into active and inactive disease activity groups based on Systemic Lupus Erythematosus Disease Activity Index (SLEDAI) score and compared the serum DNAase1 level between the two groups.

Results Out of 33 children enrolled, 13 (39.3\%) had active disease (SLEDAI score 3) and $20(60.6 \%)$ had inactive disease activity. Mean age at diagnosis was 8.5 years and 10.2 years in active and inactive groups respectively. There is female preponderance $(66.7 \%)$ in the enrolled patients. Anti nuclear antibody (ANA) was positive in $90.9 \%$ of patients. The most common pattern of ANA was diffuse pattern (48.4\%). The patients in active disease activity group presented most commonly with nephritis (53.8\%), rash (53.8\%), arthralgia (38.5\%), oral ulcer (30.8\%) and central nervous system (CNS) involvement (38.4\%) while patients in inactive disease activity group presented with nephritis (35\%), arthralgia (35\%), rash (25\%) and CNS involvement (30\%). Class III/IV lupus nephritis was present in $25 \%$ in active disease activity group while it was present in $23.1 \%$ in inactive disease activity group. Anti-double stranded DNA (anti dsDNA) was elevated in $53.8 \%$ in active group and $50 \%$ in inactive group. Antiphospholipid antibody was present in $3(23.1 \%)$ in active disease activity group and $5(25 \%)$ in inactive disease activity group. The mean serum concentrations of DNAase1 were $15.394 \mathrm{ng} /$ $\mathrm{ml}$ in active disease group and $15.205 \mathrm{ng} / \mathrm{ml}$ in inactive disease group. There was no statistically significant difference in the serum DNAase1 concentrations between the two groups $(p=0.943)$. There was also no significant difference in the mean serum concentration of DNAase1 in patients with or without nephritis $(p=0.080)$.

Conclusions The present study could not established any correlation between serum DNAase1 levels and disease activity in pediatric-onset SLE. There was no association between serum DNAase1 levels and organ involvement such as nephritis in the enrolled patients.

Funding Source(s): None

\section{SEXUAL DYSFUNCTION IN A BRAZILIAN COHORT OF BELIMUMAB TREATED SLE PATIENTS}

Morton Scheinberg*, Andrea Pimentel Fonseca Golmia, Ricardo Golmia, Gislene Ferrarezi, Aline Pinheiro dos Santos Cortada. Clinical Research Center Hospital AACD

\subsection{6/lupus-2019-Ism.279}

Background Systematic review and metaanalysis results of sexual dysfunction in patients with systemic lúpus erythematosus are contradictory. We investigate the quality of sexual activity in 74 patients with SLE receiving standard care of therapy and the addition of monthly injections of belimumab.

Methods The Female Sexual Function Index (FSfi), obtained by applying a 19-item questionnaire that assesses six domains (sexual desire, arousal, vaginal lubrication, orgasm, sexual satisfaction and pain), was used. 